"C 2019 IEEE. Personal use of this material is permitted. Permission from IEEE must be obtained for all other uses, in any current or future media, including reprinting/republishing this material for advertising or promotional purposes, creating new collective works, for resale or redistribution to servers or lists, or reuse of any copyrighted component of this work in other works." 


\title{
MPTC for PMSMs of EVs With Multi-Motor Driven System Considering Optimal Energy Allocation
}

\author{
Xiaodong $\operatorname{Sun}^{1}$, Changchang $\mathrm{Hu}^{1}$, Jianguo $\mathrm{Zhu}^{2}$, Shaohua Wang ${ }^{1}$, Weiqi Zhou ${ }^{1}$, \\ Zebin Yang ${ }^{3}$, Gang $\mathrm{Lei}^{2}, \mathrm{Ke} \mathrm{Li}^{3}$, Bin $\mathrm{Zhu}^{1}$, and Youguang $\mathrm{Guo}^{2}$ \\ ${ }^{1}$ Automotive Engineering Research Institute, Jiangsu University, Zhenjiang 212013, China \\ ${ }^{2}$ School of Electrical and Data Engineering, University of Technology, Sydney, Sydney, NSW 2007, Australia \\ ${ }^{3}$ School of Electrical and Information Engineering, Jiangsu University, Zhenjiang 212013, China
}

\begin{abstract}
This paper presents a compound propulsion system with a high-speed permanent- magnet synchronous motor (PMSM) and two inwheel motors for electric vehicles (EVs). In this study, the longitudinal dynamics model of EVs is firstly presented. Then traction distribution ratio $\alpha$ is introduced to express the traction distribution between the front and the rear axle. Moreover, the function of power consumption in concerned with the traction distribution ratio $\alpha$ is established. Therefore, the $\alpha$ that minimize the power consumption function is selected as the optimal traction distribution ratio. To improve the performance of motor controllers, the model predictive torque control (MPTC) method is employed for high speed and in-wheel motor drives. Experimental comparison with field-oriented control (FOC) shows the advantages of MPTC in dynamic response. Finally, experimental comparisons and HiL tests are presented to verify the MPTC method and the proposed energy allocation method, respectively.
\end{abstract}

Index Terms-Electric vehicle, permanent magnet synchronous motor, vehicle propulsion system, model predictive torque control

\section{INTRODUCTION}

$\mathrm{W}$ ith the development of society and economy, environment deterioration and energy shortage are getting more and more concerns. Energy conservation, emission reduction and improving the efficiency of drive system under limited battery capacity have become increasingly important. However, the advantage of the motor drive system cannot be fully utilized as electric vehicles adopts the single power source structure as traditional vehicles [1], [2]. In order to improve the energy efficiency of electric vehicles, propulsion system with multiple traction motors can be adopted. Multi-motor propulsion system can be divided into two types, the four in-wheel motor distributed propulsion system and the front and rear dual motor distributed propulsion system. In [3], R. Wang conducts motor dynamics experiments using four independently driven in-wheel motors and analyzed the energy efficiency characteristics of the wheel motors based on experimental data. Then, an energy distribution method for improving the efficiency of four inwheel vehicles is proposed. In [4] and [5], N. Mutoh presents the torque distribution strategy of micro-electric vehicles driven by different types of motors in front axle and rear axle. Then the misalignment model of the motor in the constant torque and weak magnetic region are studied, respectively. This strategy can improve the efficiency of motors with different speed range. In [6] and [7], Y. Chen proposes a dualdrive system with speed and torque coupling. Aiming at optimizing the 100-km acceleration time and driving distance, quantum genetic algorithm is used to optimize the parameters of the dynamic system. As each motor in the four in-wheel motors system can be independently controlled, the dynamics

Manuscript received October 28, 2018; accepted March 6, 2019. Corresponding author: S. Wang (e-mail: wsh@ujs.edu.cn)

Color versions of one or more of the figures in this paper are available online at http://ieeexplore.ieee.org. performance of vehicle in this scheme is better than in dualmotor driven system. However, serious safety accidents may be caused, if an in-wheel motor or a motor drive fails at high speed.

In this paper, a composed propulsion system with a high speed PMSM for the front axle and two in-wheel motors for the rear axle is proposed. While, since the in-motor needs to be embedded in the wheel hub, the motor size needs to be reduced. Due to the advantages of high efficiency, high torque density, and widely speed range [8-11], the permanent-magnet synchronous motor (PMSM) is considered as a suitable type of drive motor for in-wheel motors.

The field-oriented control (FOC) method is commonly used for PMSM control. The FOC system usually utilizes cascade control loops with several PI controllers for speed, position and torque control [12], [13]. Due to the advantages of simple algorithm, good robustness and high reliability, the FOC method has been widely employed in PMSM cntrol. However, the cascade structure has its drawback in restricting the overall performance optimization control and disturbance compensation [14-18]. In order to improve the performance of motor drives, the model predictive control (MPC) approach has been developed in recent years. MPC uses mathematic methods to predict the state of the motor at next period according to the motor model, and then the voltage vector of inverter that can minize the cost function will be selected as a controller output during the next control period [19-25]. There are two types MPC methods usually adopted in motor drives, the model predictive current control (MPCC) method, and the model predictive torque control (MPTC) method. In [25], comparions between MPTC and MPCC have been carried out and the advantages of MPTC in reducing torque ripples and improving response speed have been pointed out.

In this paper, the MPTC method is employed to control inwheel motors and the high speed motor. Experimental comparisions with FOC in torque tracking and disturbance rejection conditions have been presented. Moreover, 
hardware-in-loop (HiL) tests are presented to verify the energy efficiency of the proposed propulsion system.

\section{VehicLE LONGITUdinAL DyNAMICS Model}

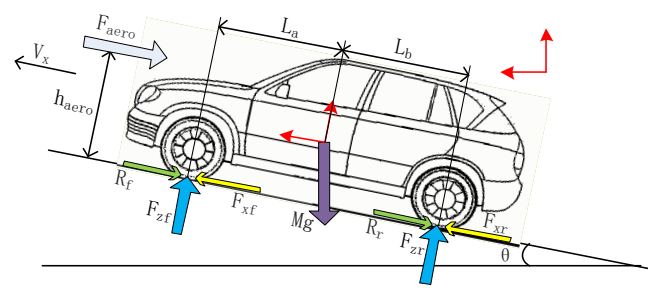

Fig. 1. Vehicle longitudinal dynamics

The torque of motors is mainly related to the load of the vehicle in the forward direction, so the longitudinal dynamics model of the vehicle is mainly concerned. As shown in Fig. 1, the vehicle is moving on a road with the slop of $\theta$ at the speed of $V \mathrm{x}$. The longitudinal dynamics of this vehicle can be described as

$$
M \stackrel{\mathrm{g}}{v_{\mathrm{x}}}=F_{x f}+F_{x r}-R_{f}-R_{r}-F_{\text {aero }}-M g \sin \theta
$$

where $F_{x f}, F_{x r}$, and $F_{\text {aero }}$ are the tire traction force of the front, the rear wheels and the aerodynamic drag force, respectively, $R_{f}$ and $R_{r}$ are the rolling resistance of the front and rear wheels, respectively, $M$ is vehicle mass; and $g$ is gravity constant. Some parameters of the vehicle are listed in table I.

Table I

\begin{tabular}{ccc}
\hline Parameters & Value & Unit \\
\hline Vehicle mass & 900 & $\mathrm{Kg}$ \\
Wheelbase & 2.2 & $\mathrm{~m}$ \\
Wheel radius & 0.275 & $\mathrm{~m}$ \\
Transmission ratio & 4.75 & \\
\hline
\end{tabular}

The aerodynamic drag force $F_{\text {aero }}$ can be expressed as

$$
F_{\text {aero }}=0.5 \rho_{d} C_{d} A_{F}\left(V_{x}+V_{\text {wind }}\right)^{2}
$$

where $\rho_{d}$ is the density of air, $C_{d}$ is the aerodynamic drag coefficient, $A_{F}$ is the frontal area of the vehicle, and $V_{\text {wind }}$ is the wind velocity in the opposite direction of vehicle velocity $V x$.

The dynamic equation of a single wheel is expressed as:

$$
I_{W} \&_{,, r}=T_{f, r}-T_{b}-R_{0} F_{x f, x r}-M_{R}-\zeta \omega_{f, r}
$$

where $I_{\mathrm{w}}$ is the tire rotational moment of inertial, $\omega_{f, r}$ is the front or rear tire rotational speed, $T_{f, r}$ and $T_{\mathrm{b}}$ represent traction torque and braking torque of front wheel or rear wheel, respectively, $F_{x f, x r}$ is the longitudinal tire-road friction force, $M_{R}$ is the moment of rolling resistance, and $\zeta$ is the damping coefficient. Since the torque of the front wheel motor is transmitted to the wheels through the transmission system with a ratio of $i_{0}$, the torque of the high speed PMSM is

$$
T_{f}{ }^{\prime}=\frac{T_{f}}{i_{0}}
$$

The proposed propulsion system is shown in Fig. 2, and its control system is shown in Fig. 3.

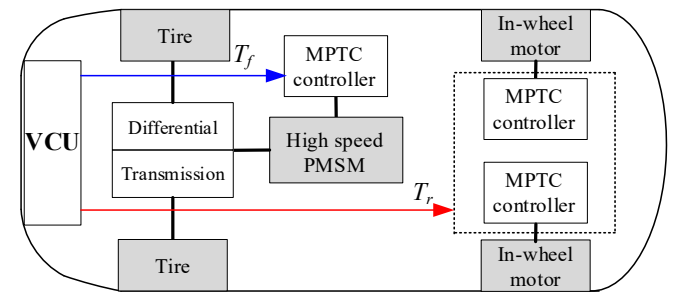

Fig. 2 Distributed propulsion system

\section{VEHICLE CONTROLLER}

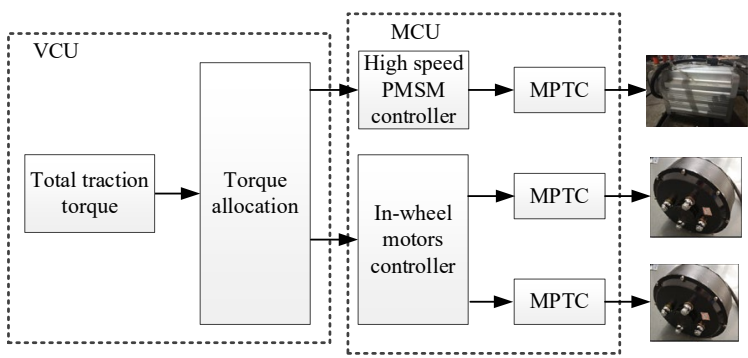

Fig. 3 Block diagram of the propulsion control system

As shown in Fig .3, The traction force $F_{x}$ can expressed as

$$
\left\{\begin{array}{l}
F_{x}=F_{x f}+F_{x r} \\
F_{x f}=\alpha \cdot F_{x}=\frac{T_{f} i_{0}}{R_{0}} \\
F_{x r}=(1-\alpha) \cdot F_{x}=2 \frac{T_{r}}{R_{0}}
\end{array}\right.
$$

where $\alpha$ and (1- $\alpha)$ denote the traction force distribution ratios of the front high speed PMSM and rear in-wheel motors, respectively. Two in-wheel motors are considered under the same traction condition.

The total instantaneous power consumption of three motors can generally be expressed as

$$
\begin{aligned}
P_{\text {total }} & =P_{f}+2 P_{r}=\frac{\omega_{f} \cdot T_{f}}{\eta_{f}\left(\omega_{f}, T_{f}\right)}+\frac{2 \omega_{r} \cdot T_{r}}{\eta_{r}\left(\omega_{r}, T_{r}\right)} \\
& =\frac{F_{x f} \cdot V_{x}}{\eta_{f}\left(V_{x}, F_{x f}\right)}+\frac{F_{x r} \cdot V_{x}}{\eta_{r}\left(V_{x}, F_{x r}\right)} \\
& =F_{x} \cdot V_{x}\left(\frac{\alpha}{\eta_{f}}+\frac{1-\alpha}{\eta_{r}}\right) \\
& =P_{\text {total }}\left(F_{x}, V_{x}, \alpha\right)
\end{aligned}
$$

where $\eta_{f}\left(\omega_{f}, T_{f}\right)$ and $\eta_{r}\left(\omega_{r}, T_{r}\right)$ stand for the efficiency of the high speed PMSM and in-wheel motors at their respective operation points, respectively.

It should be noted that, the total power consumption of the propulsion system is determined by total traction force $F_{x}$, vehicle speed $V_{x}$, and the traction force distribution ratio $\alpha$. Therefore, the efficiency of the multi-motor drivn system can be optimized by selecting an appropriate $\alpha$ value for each operation point $\left(F_{\mathrm{x}}, V_{\mathrm{x}}\right)$, as described in (7)

$$
\alpha^{*}=\arg \min \left\{P_{\text {total }}\left(F_{x}, V_{x}, \alpha\right) \mid 0 \leq \alpha \leq 1\right\}
$$

s.t. 


$$
\left\{\begin{array}{l}
F_{\mathrm{xf}}=\alpha \cdot F_{\mathrm{x}} \leq T_{\mathrm{f}, \max }\left(\frac{V_{\mathrm{x}} \cdot i_{0}}{R_{0}}\right) \cdot \frac{i_{0}}{R_{0}} \\
F_{\mathrm{xr}}=(1-\alpha) \cdot F_{\mathrm{x}} \leq T_{\mathrm{r}, \max }\left(\frac{V_{\mathrm{x}}}{R_{0}}\right) \cdot \frac{2}{R_{0}} \\
0 \leq V_{\mathrm{x}} \leq V_{\text {max }}
\end{array}\right.
$$

The optimal $\alpha$ can thus be obtained by solving the above equation as shown in Fig.4

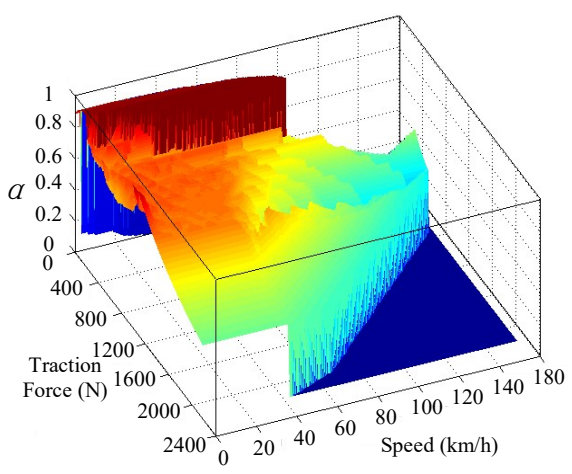

Fig. 4 Optimum traction distribution ratio at different torque and speeds

\section{MOTOR CONTROLlER}

\section{A. Dynamic Equations of PMSM}

Different from traditional single-motor-driven electric vehicles, the multi-motor-driven electric vehicle can distribute the traction torque more efficiently. Therefore, the motor is required to have a higher response speed. Model predictive control is widely recognized as a high-performance control strategy of PMSM drives due to its quick response and simple principle. While MPTC shows that it is more superior than MPCC at improving system dynamic characteristics.

The model of a surface maintained PMSM in $d q$-axis rotor frame can be written as

$$
\left\{\begin{array}{l}
u_{d}=R_{s} i_{d}-\omega_{e} \psi_{q}+\frac{d \psi_{d}}{d t} \\
u_{q}=R_{s} i_{q}+\omega_{e} \psi_{d}+\frac{d \psi_{q}}{d t}
\end{array}\right.
$$

the stator flux equation are

$$
\left\{\begin{array}{l}
\psi_{d}=L i_{d}+\psi_{f} \\
\psi_{q}=L i_{q}
\end{array}\right.
$$

where $u_{d}, u_{q}, i_{d}, i_{q}, \psi_{d}, \psi_{q}$ are the voltages, currents and magnetic flux of stator in $d q$-axis, respectively. $R_{s}$ and $L$ represent the stator resistance and inductance, respectively. $\omega_{e}$ is electromagnetic angular velocity, and. $\psi_{f}$ is the permanent magnet flux linkage.

A discrete-time model of a surface mounted PMSM is described as follow:

$$
\left\{\begin{array}{l}
\psi_{s}^{k+1}=T_{s} u^{k}+\psi_{s}^{k}-T_{s} i_{s}^{k} R_{s} \\
T_{m}^{k+1}=\frac{3}{2} P_{p} \psi_{m} i_{s}^{k+1}
\end{array}\right.
$$

Thus, the flux linkage and the torque can be calculated directly by obtaining the stator current at time $k+1$. As follows:

$$
i_{s}^{k+1}=i_{s}^{k}+\frac{T_{s}}{L_{s}}\left(u_{s}^{k}-R_{s} i_{s}^{k}-j \omega \psi_{f} e^{j \theta}\right)
$$

The estimation value of $\psi_{s}^{k+1}$ and $T_{m}^{k+1}$ are utilized for the prediction process. The voltage $u_{q}^{k}$ is implemented by the inverter at sampling instant $k$.

\section{B. Principle of MPTC}

While in practical implementation, due to various delay of digital control system, the selected controller output can not be applied to the system until the next instant. This phenomenon will degrade the control performacne of the system especially when the sampling frequency is low. Therefore, it is necessary to compensate the impact of one-step delay.

Stator current at time $k+1$ can be calculated from equation (11), and when it is used to calculate torque and flux instead of the current $i_{s}{ }^{k}$, the delay impact will be compensated. However, in order to compensate the one-step delay more effectively, the accuracy of current prediction need to be improved. An improved Euler discretization algorithm is applied

$$
\left\{\begin{array}{l}
i_{p}^{k+1}=i_{s}^{k}+\frac{T_{s}}{L_{s}}\left(u_{s}^{k}-R_{s} i_{s}^{k}-j \omega \psi_{f} e^{j \theta}\right) \\
i_{s}^{k+1}=i_{p}^{k+1}+\frac{-R_{s} T_{s}\left(i_{p}^{k+1}-i_{s}^{k}\right)}{2 L_{s}}
\end{array}\right.
$$

The cost function is usually concerned with control targets. In EVs, motor controllers are supposed to minize the flux and electromagnetic torque tracking error.

$$
J=\left|T_{m}^{*}-T_{m}^{p}\right|+Q\left|\psi_{s}^{*}-\psi_{s}^{p}\right|
$$

where $Q$ is a weighting factor that represents the importance of flux linkage tracking in comparison to torque tracking. For a two-level inverter, there are only eight voltage vectors (including two null vectors). The best voltage vector that minize the above cost function will be applied in the next control period. The block diagram of proposed MPTC is shown in Fig.5.

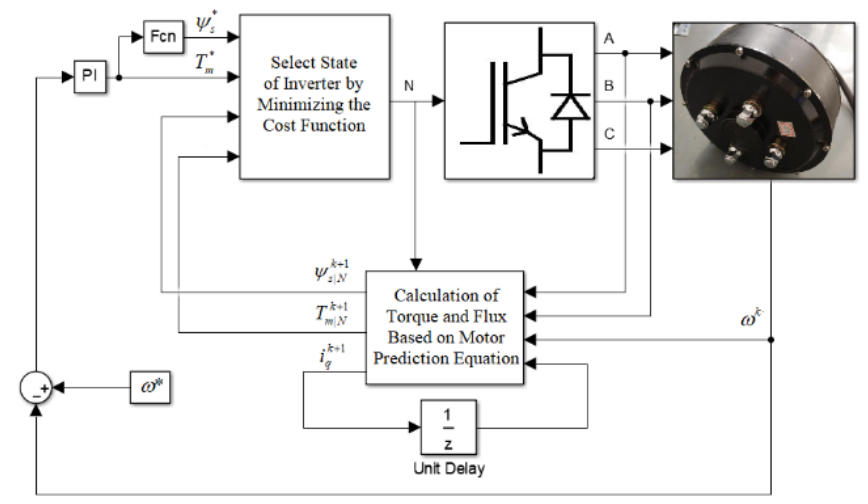

Fig. 5 Block diagram of MPTC

\section{V.EXPERIMENTS}

To validate the performance of MPTC controller, 
comparision experiments with traditional field oriented control (FOC) method have been carried out. Experimental setups shown in Fig. 6 and Fig. 7 are used to test in-wheel motor controller and high speed PMSM controller, respectively. The test bench consisits of a dSPACE DS1401 PPC, a torque sensor, and a magnetic power brake. In order to faciliate the comparision, in-wheel motor controllers and high speed PMSM controller are set to cope with the same traction torque conditions.

First, the vehicle starts acclerating at $0.3 \mathrm{~s}$ with an acceleration of $2 \mathrm{~m} / \mathrm{s}^{2}$, and the resistance variation are ignored and $\alpha$ is set to 0.6 . And the traction torque of high speed PMSM and in-wheel motor can be calculated from (5)

$$
\left\{\begin{array}{l}
F_{X}=M a=1750 \mathrm{~N} \\
T_{f}=\frac{R_{0}}{i_{0}} \alpha F_{x}=60.8 \mathrm{~N} \cdot \mathrm{m} \\
T_{r}=\frac{R_{0}}{2}(1-\alpha) F_{x}=96.25 \mathrm{~N} \cdot \mathrm{m}
\end{array}\right.
$$

Dynamic response of in-wheel motor and high speed PMSM in acceleration condition for MPTC method and FOC method are recorded in Fig. 8.

Next, the motor controllers are tested against sudden external disturbance variations. That is, after the torque of the high speed PMSM and in-wheel motor is stable, a external torque of $10 \mathrm{~N} \cdot \mathrm{m}$ for $0.1 \mathrm{~s}$ is applied to them. The torque response under this condition are presented in Fig.9 for MPTC method and FOC method.

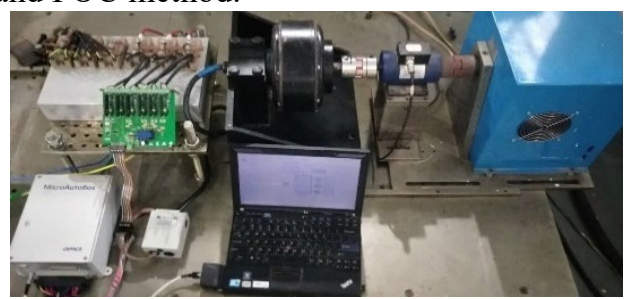

Fig. 6 Experimental setup for in-wheel motor

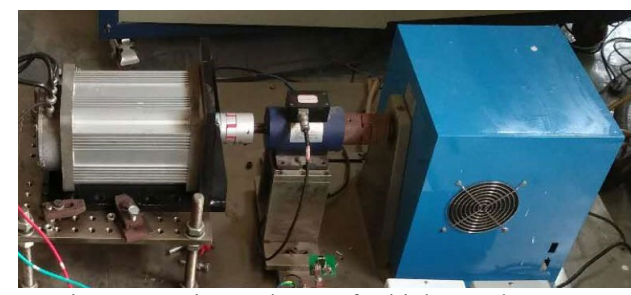

Fig. 7 Experimental setup for high speed PMSM

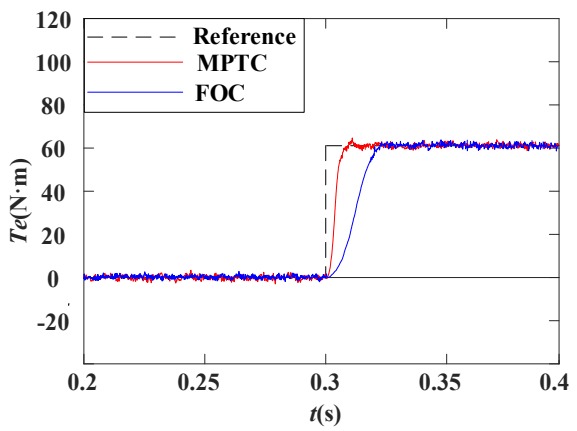

(a)

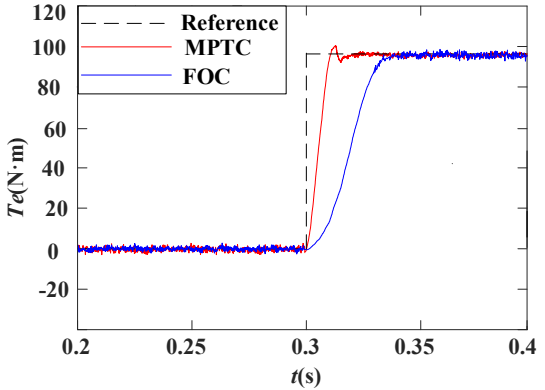

(b)

Fig. 8 Experimental results of torque dynamics in acceleration condition (a) Inwheel motor (b) High speed PMSM

Figs. 8 and 9 show the torque response comparisions of the conventional FOC and MPTC under acceleration condition and external disturbance condition, respectively. As can be observed from Fig. 8, when the reference torque is changed in $0.3 \mathrm{~s}$, the MPTC in-wheel motor drive takes $0.012 \mathrm{~s}$ to adjust, while the settling time of FOC in-wheel motor drive is around $0.023 \mathrm{~s}$. It can also be noted that, the MPTC high speed PMSM drive can reduce $50 \%$ time for adjustment compared to the FOC high speed PMSM drive. As shown in Fig.9, when external disturbance occurs, the MPTC drive can not only reduce the settling time, but also supress overshoot more effectively. The advantage of MPTC in fast response can improve the acceleration performance of the vehicel directly. Morever, as $\alpha$ changes according to working conditions, MPTC can help the motor drives to implement the torque distribution strategy more effectively, and the energy consumption can thus be reduced.

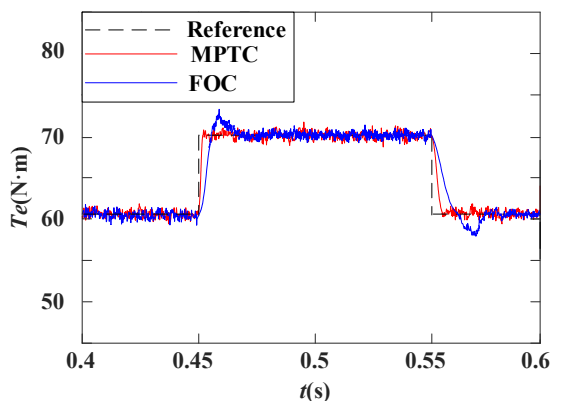

(a)

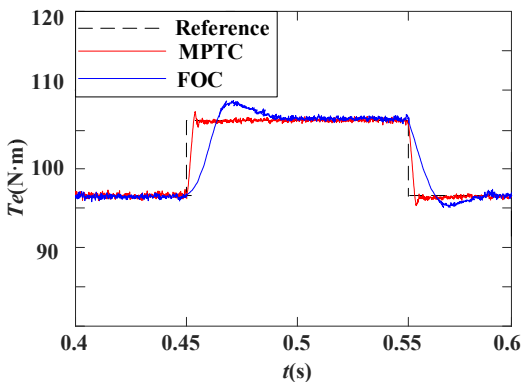

(b)

Fig. 9 Experimental results of torque dynamics in external disturbance condition (a) In-wheel motor (b) High speed PMSM

Parameter perturbation and permanent magnet demagnetization are difficult to simulate, thus HiL test is performed to verify the performance of the proposed traction allocation method for the compound EV propulsion system. 
HiL test setups are shown in Fig. 10. In addition, the simulink model of the vehicle dynamics and battery management system (BMS) are also combined for energy management.

In order to highlight the superiority of the proposed traction allocation method in energy efficiency, comparision tests in the Urban Dynamometer Driving Schedule (UDDS) with a evenly distributed method have been carried out. With the evenly distributed method, the traction force distribution ratio $\alpha$ is 0.5 , i.e., $F_{x f}=F_{x r}=0.5 F_{x}$.

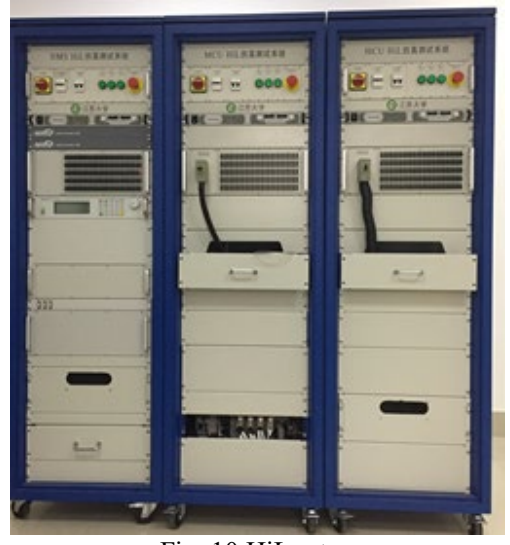

Fig. 10 HiL setup

Fig. 11 shows the changes of SOC during UDDS. It can be noted that after 5 UDDS, the proposed energy allocation method can save more than $6 \%$ energy than the evenly distributed method.

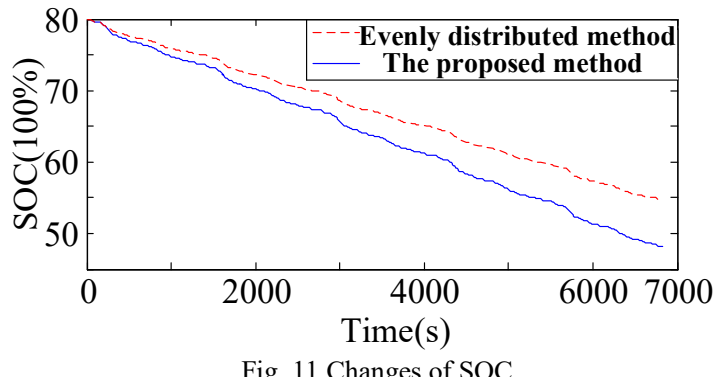

\section{CONCLUSION}

This paper presents a MPTC method for a multi-motor driven EV with a high speed PMSM and two in-wheel PMSMs. Moreover, the optimal energy allocation is also considered in this study. The optimal force distribution ratio changes according to the driving condition. Thus, a quick respose of motor is required. Experimental tests show that the superiority of MPTC in response speed compared to traditional FOC method. Finally, the HiL tests are carried to verify the advantage of the proposed energy allocation method in energy efficiency compared with the evenly distributed method.

\section{ACKNOWLEDGEMENT}

This work was supported by the National Natural Science Foundation of China under Project 51875261, the Natural Science Foundation of Jiangsu Province of China under Projects BK20180046 and BK20170071, the "Qinglan project" of Jiangsu Province, the Key Project of Natural Science Foundation of Jiangsu Higher Education Institutions under Project 17KJA460005, the Six Categories Talent Peak of Jiangsu Province under Projects 2015-XNYQC-003 and 2016-GDZB-096, the "333 project" of Jiangsu Province under Project BRA2017441, the Postgraduate Research \& Practice Innovation Program of Jiangsu Province under Project SJCX18_0745.

\section{REFERENCES}

[1] Z. Wang, et al., "Dynamic control of motor torque during the shifting process in PMSM-AMT system." IEEE Transportation Electrification Asia-Pacific, vol. 60, no. 4 pp. 1-5, 2014.

[2] X. Sun, Y. Shen, S. Wang, G. Lei, Z. Yang, and S. Han, "Core losses analysis of a novel 16/10 segmented rotor switched reluctance BSG motor for HEVs using nonlinear lumped parameter equivalent circuit model", IEEE/ASME Trans. on Mechatron., vol. 23, no. 2, pp. 747-757, 2011.

[3] R. Wang, Y. Chen, D. Feng, X. Huang, and J. Wang, "Development and performance characterization of an electric ground vehicle with independently actuated in-wheel motors," J. Power Sources, vol. 196, no. 8, pp. 3962-3971, Apr. 2011.

[4] N. Mutoh, T. Horigome, and K. Takita, "Driving characteristics of an electric vehicle system with independently driven front and rear wheels," IEEE Trans. Ind. Electron., vol. 53, no. 3, pp. 803-813, Jun. 2006.

[5] N. Mutoh and Y. Nakano, "Dynamics of front-and-rear-wheelindependent-drive-type electric vehicles at the time of failure," IEEE Trans. Ind. Electron., vol. 59, pp. 1488-1499, 2012.

[6] Y. Chen and J. Wang, "Energy-efficient control allocation with applications on planar motion control of electric ground vehicles," Proc. Amer. Control Conf., San Francisco, pp. 2719-2724, 2011,

[7] Y. Chen and J. Wang, "Fast and global optimal energy-efficient control allocation with applications to over-actuated electric ground vehicles" IEEE Trans. Control Syst. Technol., vol. 20, no. 5, pp. 1202-1211, Aug. 2011.

[8] M. Zeraoulia, M. E. H. Benbouzid, and D. Diallo, "Electric motor drive selection issues for HEV propulsion systems: A comparative study," IEEE Trans. Veh. Technol., vol. 55, no. 6, pp. 1756-1764, Nov. 2006.

[9] X. Sun, Z. Jin, S. Wang, Z. Yang, K. Li, Y. Fan and L. Chen. Performance improvement of torque and suspension force for a novel five-phase BFSPM machine for flywheel energy storage systems. IEEE Trans. on Appl. Supercon., vol. 29, no. 2, Art. no.: 0601505, 2019.

[10] Z. Shi, X. Sun, Y. Cai, Z.Yang, G. Lei, Y. Guo, and J. Zhu. Torque analysis and dynamic performance improvement of A PMSM for EVs by skew angle optimization. IEEE Trans. on Appl. Supercon., vol. 29, no. 2, Art. no.: 0600305, 2019.

[11] X. Sun, Z. Shi, L. Chen, and Z. Yang, "Internal model control for a bearingless permanent magnet synchronous machine based on inverse system method," IEEE Trans. on Energy Conversion, vol. 31, no. 4, pp. 1539-1548, 2016.

[12] X. Sun, L. Chen, Z. Yang, and H. Zhu, "Speed-sensorless vector control of a bearingless induction motor with artificial neural network inverse speed observer", IEEE/ASME Trans. on Mechatronic., vol. 18, no. 4, pp. 1357-1366, 2013.

[13] Y. P. Yang and D. S. Chuang, "Optimal design and control of a wheel motor for electric passenger cars," IEEE Trans. Magn., vol. 43, no. 1, pp. 51-61, Jan. 2007.

[14] S. Sue and C. Pan, "Voltage-Constraint-Tracking-Based FieldWeakening Control of IPM Synchronous Motor Drives," IEEE Trans. Ind. Electron., vol. 55, no. 1, pp. 340-347, Jan. 2008.

[15] X. Sun, B. Su, S. Wang, Z., G. Lei, J. Zhu, and Y. Guo, "Performance analysis of suspension force and torque in an IBPMSM with V-shape PMs for flywheel batteries," IEEE Trans. on Magnetic., vol. 54, no. 11, Art. no.: 8105504, 2018.

[16] X. Sun, L. Chen, H. Jiang, Z. Yang, J. Chen, and W. Zhang. "Highperformance control for a bearingless permanent magnet synchronous motor using neural network inverse scheme plus internal model controllers." IEEE Trans. on Ind. Electron., vol. 63, no. 6, pp. 34793488, 2016.

[17] M. Preindl and S. Bolognani, "Model predictive direct speed control with finite control set of PMSM drive systems," IEEE Trans. Power 
Electron., vol. 28, no. 2, pp. 1007-1015, Feb. 2013.

[18] B. Boazzo and G. Pellegrino, "Model-Based Direct Flux Vector Control of Permanent-Magnet Synchronous Motor Drives," IEEE Trans. Ind. App, vol. 51, no. 4, pp. 3126-3136, July-Aug. 2015.

[19] M., H., et al., "A Simplified Finite-State Predictive Direct Torque Control for Induction Motor Drive." IEEE Trans. Ind. Electron., vol. 63, no. 6, pp. 3964-3975, 2016.

[20] Y. Zhang and W. Xie, "Low Complexity Model Predictive ControlSingle Vector-Based Approach," IEEE Trans. Power Electron., vol. 29, no. 10, pp. 5532-5541, Oct. 2014.

[21] Y. Zhang and H. Yang, "Generalized Two-Vector-Based ModelPredictive Torque Control of Induction Motor Drives," IEEE Trans. Power Electron., vol. 30, no. 7, pp. 3818-3829, July 2015.

[22] Y. Zhang, D. Xu, J. Liu, S. Gao and W. Xu, "Performance Improvement of Model-Predictive Current Control of Permanent Magnet Synchronous Motor Drives," IEEE Trans. Ind. App., vol. 53, no. 4, pp. 3683-3695, July-Aug. 2017.

[23] Q. Liu and K. Hameyer, "Torque Ripple Minimization for Direct Torque Control of PMSM With Modified FCSMPC," IEEE Trans. Ind. App., vol. 52, no. 6, pp. 4855-4864, Nov.-Dec. 2016.

[24] N. K. Adamopoulos, , et al., "Comparison of field oriented versus model predictive torque control techniques for monitoring interior PM traction motor over wide speed range," 2017 IEEE 11th International Symposium on Diagnostics for Electrical Machines, Power Electronics and Drives (SDEMPED), Tinos, 2017.

[25] Geyer, T, "A comparison of control and modulation schemes for medium-voltage drives: emerging predictive control concepts versus PWM-based schemes," IEEE Trans. Ind. Appl., vol. 47, no. 3, pp. 1380-1389, 2011. 